\title{
BMJ Open Discontinuing Inappropriate Medication in Nursing Home Residents (DIM-NHR Study): protocol of a cluster randomised controlled trial
}

\author{
Hans Wouters, ${ }^{1}$ Elise H Quik, ${ }^{1}$ Froukje Boersma, ${ }^{2}$ Peder Nygård, ${ }^{3}$ Judith Bosman, ${ }^{3}$ \\ Wendelien M Böttger, ${ }^{3}$ Hans Mulder, ${ }^{4}$ Jan-Gerard Maring, ${ }^{3}$ Linda Wijma-Vos, ${ }^{5}$ \\ Tim Beerden, ${ }^{5}$ Jasperien van Doormaal, ${ }^{5}$ Maarten J Postma, ${ }^{6}$ Sytse U Zuidema, ${ }^{2}$ \\ Katja Taxis ${ }^{1}$
}

To cite: Wouters H, Quik EH, Boersma F, et al. Discontinuing Inappropriate Medication in Nursing Home Residents (DIM-NHR Study): protocol of a cluster randomised controlled trial. BMJ Open 2014;4:e006082. doi:10.1136/bmjopen-2014006082

- Prepublication history for this paper is available online. To view these files please visit the journal online (http://dx.doi.org/10.1136/ bmjopen-2014-006082).

Received 9 July 2014 Accepted 21 August 2014

CrossMark

For numbered affiliations see end of article.

Correspondence to Dr Hans Wouters; h.wouters@rug.nl

\section{ABSTRACT}

Introduction: Nursing home residents often have a high number of comorbidities resulting in polypharmacy. Inappropriate prescribing is therefore likely to occur, which in turn is expected to worsen cognitive impairment, to increase the fall risk and to decrease residents' quality of life. The objective of the 'Discontinuing Inappropriate Medication in Nursing Home Residents' (DIM-NHR) study is to examine the efficacy and cost-effectiveness of the Multidisciplinary Multistep Medication Review (3MR) that is aimed at optimising prescribing and discontinuing inappropriate medication.

Methods: A cluster randomised controlled trial will be conducted. Elderly care physicians and their wards (clusters) will be randomised. Data will be collected at baseline and 4 months after the 3MR has taken place. Six hundred nursing home residents will be recruited of whom more than half are expected to suffer from dementia. The 3MR will be based on consensus criteria and the relevant literature and will be performed by the patient's elderly care physician in collaboration with a pharmacist.

Analysis: Primary outcomes - the difference in proportion of residents who successfully discontinued inappropriate medication between the intervention and control group at follow-up. Secondary outcomesundertreatment, exposure to anticholinergic and sedative medicines, neuropsychiatric symptoms, cognitive function, falls, hospital admission, quality of life and cost-effectiveness.

Ethics and dissemination: Participant burden will be kept at a minimum. The elderly care physician will remain free to adjust medication when symptoms relapse or adverse events occur, rendering serious adverse events highly unlikely. Study findings will be published in peer-reviewed journals and a 3MR toolkit will be developed.

Trial registration number: This study has been registered at http://www.ClinicalTrials.gov (trial registration number: NCT01876095)

\section{INTRODUCTION}

Nursing home residents are among the frailest patient groups. They often have a high number of comorbidities and are consequently prescribed a high number of medicines. Research suggests that as much as $40 \%$ of prescriptions for nursing home residents may be inappropriate or suboptimal. ${ }^{1}$ Inappropriate medication prescribing is commonly associated with adverse events, hospital admissions as well as other forms of unnecessary healthcare utilisation and mortality. $^{2-4}$ Moreover, a large proportion of nursing home residents who suffer from dementia are treated with psychotropic drugs for neuropsychiatric symptoms. This is another addition to the drug burden. Prescribing of medicines is further complicated by pharmacokinetic and pharmacodynamic changes of the ageing body. ${ }^{1}$ Thus, given its magnitude, adverse consequences and complexity, inappropriate prescribing is a major challenge to overcome in the care of nursing home residents. The need to improve prescribing is therefore urgent.

Hitherto conducted studies, aimed at discontinuing inappropriate drug prescribing, can be classified into two types. The first type of studies targeted discontinuation of specific medicines, whereas the second type examined sensible discontinuation of an array of medicines guided by medication reviews. Evidence from both types of studies suggests that discontinuation of overprescribed medication does not necessarily result in declined health states and may even improve health outcomes. The first type of studies showed, for instance, that discontinuation of antidepressants was tolerated in patients with 
dementia. ${ }^{5}$ Furthermore, a systematic review of withdrawal of antihypertensive drugs in elderly patients showed that $20-85 \%$ of the patients remained normotensive, or did not require reinstatement of therapy without increase in mortality. ${ }^{6}$ Although there is evidence of the effectiveness of treating hypertension in older people who are fit, in the frailer populations overenthusiastic attempts to lower blood pressure have been associated with increased mortality and morbidity. ${ }^{7}$ Another class of medicines that are worthwhile to discontinue are antipsychotics. Antipsychotics have only limited efficacy for treatment of neuropsychiatric symptoms in patients with dementia but are associated with considerable side effects such as accelerated cognitive decline, ${ }^{8}$ risk of stroke and death. ${ }^{9}$ Although discontinuation of antipsychotics is advocated in all guidelines and most discontinuation studies did not find a relapse of neuropsychiatric symptoms,${ }^{10}$ one recent study actually found a relapse of neuropsychiatric symptoms. ${ }^{11}$ Because of this relapse of neuropsychiatric symptoms, it may not be possible to discontinue antipsychotics for every patient.

The second type of studies has looked at the value of (multidisciplinary) systematic medication reviews by a physician in collaboration with a pharmacist. In a small non-randomised study on discontinuation of multiple medicines in an Israeli nursing home population, physicians stopped medication in $63 \%$ of patients and subsequently only $10 \%$ of the medicines had to be reinstated. Mortality and referrals to hospitals significantly decreased and there were savings on drug costs. Physicians used the so called Good Palliative-Geriatric Practice algorithm for drug discontinuation as a tool to guide the medication review process. ${ }^{12}$ There is some evidence on the efficacy of medication reviews from other small and less rigorously conducted studies as well. And there is also further evidence that multidisciplinary medication reviews save costs. ${ }^{13}$ A recent cluster randomised controlled trial (RCT) in nursing homes in Northern Ireland by Patterson et $a l^{14}{ }^{15}$ showed that medication reviews were successful in reducing inappropriate psychotropic medication use compared with usual care and that these were cost-effective as well. Furthermore, a recent systematic review on medication reviews and other related interventions to improve prescribing in nursing home residents showed conflicting results. ${ }^{16}$ However, several studies had methodological limitations. These included inappropriate outcomes studied, small sample size and the medication review being too non-committal as well as the heterogeneity of the design of medication reviews. ${ }^{15-17}$ It is therefore difficult to determine whether the contradicting findings reflect actual differences between studies or stem from mere methodological shortcomings. Furthermore, outcomes such as harm and hospital admission, cognitive impairment and neuropsychiatric symptoms might be more convincing, but so far the use of such outcomes has been limited; or only small effects on these measures have been found. Moreover, except from the cluster
RCT by Patterson et al, ${ }^{14}$ little is known about the costeffectiveness of systematic medication reviews. Taken together, there remains a substantial knowledge gap with respect to the efficacy and cost-effectiveness of systematic medication reviews, especially in the nursing home setting.

In our study, systematic medication reviews will be carried out by the treating elderly care physician in close collaboration with the pharmacist. These medication reviews will be aimed at optimising prescribing, in particular by focusing on discontinuing inappropriate medication but also by considering undertreatment by starting appropriate medication that was lacking.

If successful, the intervention will lead to an optimisation of prescribing in nursing home residents. On the one hand, the number of medicines the nursing home residents are taking will be reduced, removing medication that is no longer necessary or has been prescribed inappropriately. This will reduce exposure to anticholinergic and sedative drug burden (as measured by the Drug Burden Index (DBI $)^{18}$-see Methods section) and associated adverse drug events, harm and number of hospital admissions, neuropsychiatric symptoms and cognitive decline, possibly increasing nursing home residents' quality of life, and will also decrease medication costs. On the other hand, necessary medication that was actually lacking will be started. This too has the potential to reduce the number of hospital admissions, and has thereby the potential to improve nursing home residents' quality of life.

As little is known about the views and opinions of the health professionals, nursing home residents and their relatives with regard to discontinuing medication in the nursing home setting, we will also examine their views and opinions qualitatively while conducting the cluster RCT. Views and opinions are, for instance, that elderly care physicians feel pressured by nursing staff to prescribe antipsychotics for behavioural problems in nursing home residents with dementia. ${ }^{19}$ Once prescribed, these medications are likely to be continued. ${ }^{20}$ Also, nursing home residents or their relatives may object to discontinuing medication because of fear of return of symptoms or development of withdrawal symptoms, but they may also welcome a reduction in the drug burden. Furthermore, it may be impossible to involve residents with severe cognitive impairment in prescribing decisions, but some residents (and their representatives) may be capable of being involved in decision-making about medication. ${ }^{21}$ Together, our study findings will provide the rationale for how to optimise prescribing in nursing home residents in order to attain optimal benefit as well as cost-effectiveness.

Accordingly, the objectives of this study proposal are (1) to examine whether multidisciplinary medication reviews effectively optimise prescribing by considering overprescribing and underprescribing; (2) whether by doing so, they reduce harm in nursing home residents and (3) whether multidisciplinary medication reviews are cost-effective. 


\section{METHODS AND ANALYSIS}

A pragmatic cluster RCT will be conducted in a manner that is consistent with the 'Consolidated Standards of Reporting Trials (CONSORT)' statement (http://www. consort-statement.org) and the 'Standard Protocol Items: Recommendations for Interventional Trials (SPIRIT)' criteria (http://www.spirit-statement.org). In each participating nursing home, elderly care physicians and their wards with residents (clusters) will be randomly allocated to the intervention versus control condition. The intervention condition will consist of the Multidisciplinary Multistep Medication Review (3MR) carried out by the patient's treating elderly care physician and a pharmacist (see Intervention section). The control condition consists of usual care including medication safety monitoring and ad hoc medication reviews on clinical indication that differs in quality and frequency, but no standardised 3MRs in the manner done in the intervention condition. Our trial can be considered a superiority trial as outcomes in favour of the intervention arm are to be expected. Outcome measures will be collected at baseline and at 4-month follow-up after the medication review.

\section{Participants}

Our ultimate aim is to enrol $\sim 600$ residents in total (see below for sample size calculation). Data collection was started on 1 June 2014 and baseline data have been collected from $\sim 35$ nursing home residents so far. To reach the target sample size, the researchers will ask for participation from a total of 30 wards and will closely monitor data collection. We estimate that half of the participants will have dementia and half will have physically disabling diseases. We adopt the following inclusion and exclusion criteria.

\section{Nursing home wards}

\section{Inclusion criteria}

- Long stay wards;

- Commitment to perform a 3MR in the manner outlined below.

Exclusion criteria

- Short stay and revalidation wards because including these will inflate the rate of nursing home residents who are lost to follow-up.

- Specialised wards for an atypical aetiology, for example, lifespan psychiatric illness or mental disability.

- Elderly care physicians who have recently received or who are to receive recertification at short notice with regard to systematic medication review methodology.

- Participation of wards in other studies aimed at improving the quality of drug prescription (in the past 12 months).

\section{Nursing home residents}

\section{Inclusion criteria}

- A life expectancy of $>4$ weeks, as judged by the treating elderly care physician.
Exclusion criteria

- Refusal of treatment with medicines;

- Having received a multidisciplinary systematic medication review in the past 6 months.

\section{Sample size calculation}

Sample size calculation is based on the primary outcome, which is the proportion of residents for whom $\geq 1$ inappropriate medications can be successfully discontinued (see Study parameters section). Based on the literature and our own pilot studies, we expect $\sim 20 \%$ of the nursing home residents will have inappropriate medication successfully discontinued when adopting usual care. We further expect that this number will increase to $\sim 40 \%$ after a 3MR in the intervention group. ${ }^{12} 1522$ Assuming 5\% significance, $80 \%$ power and an intracluster correlation coefficient of $0.1,{ }^{23}$ requires participation by a total of 420 residents or 28 elderly care physicians ( 14 in each condition) $\times 15$ nursing home residents. To allow for $\sim 30 \%$ attrition of residents, we will recruit $\sim 600$ residents or 30 elderly care physicians times $\sim 20$ residents.

\section{Intervention}

The 3MR will be carried out by the elderly care physicians in close collaboration with a pharmacist. It is an optimised approach for systematic and periodical reviewing of medication (see table 1). The medication review process will be piloted by a pharmacist and an elderly care physician who do not participate in the study.

\section{Rationale for intervention steps}

Steps 1 and 2 concerning 'Patient involvement \& Exploration' and 'Drug Reviewing', respectively (see table 1), provide a good preparation of the actual medication review in step 3, the 'Multidisciplinary Meeting', which is essential to let the medication review succeed. ${ }^{17}$ Moreover, step 1 incorporates the patient perspective. ${ }^{29}$ We ask the elderly care physician in step 1 to provide the pharmacist with a list of diagnoses and actual problems of the nursing home residents. On clinical indication, the elderly care physician will consult a medical specialist (neurologist, geriatric psychiatrist, geriatrician, cardiologist) to verify the diagnoses. In step 2, the pharmacist will adopt the standardised STOPP and START criteria to identify potential underprescribing/ misprescribing and overprescribing. ${ }^{24} \quad 30$ Step 3 is important in order to attain multidisciplinary consensus about optimal recommendations, which prevents the medication review from becoming a mere single direction advice given by the pharmacist. ${ }^{17} 30$

Steps 4 and 5, concerning the 'Pharmacotherapeutical Action Plan' and the 'Implementation \& Interim Evaluation Action Plan', respectively, are important in order to initiate action following the medication review, to facilitate clear communication and mutual feedback about all arrangements and appointments for the elderly care physician and a pharmacist, and to involve 


\begin{tabular}{|c|c|c|c|}
\hline Step & Time & Initiator & Label and description \\
\hline \multirow[t]{2}{*}{$\# 1$} & $10 \mathrm{~min}$ & ECP & Patient involvement \& Exploration \\
\hline & & NS & $\begin{array}{l}\text { Nursing home residents' experiences and preferences will be assessed by the responsible } \\
\text { member of the NS using a standardised questionnaire. Experiences and preferences of } \\
\text { incapacitated nursing home residents will be assessed through a questionnaire filled out by the } \\
\text { patient's representative. Furthermore, in preparation of step \#2, the ECP will compile the patient's } \\
\text { diagnoses, allergies and laboratory results. On clinical indication, the ECP will consult a medical } \\
\text { specialist to verify the diagnosis. Subsequently, the ECP will send these data together with the } \\
\text { questionnaire to the P }\end{array}$ \\
\hline \multirow[t]{2}{*}{ \#2 } & $10 \mathrm{~min}$ & $\mathrm{P}$ & Drug Reviewing \\
\hline & & & $\begin{array}{l}\text { The } P \text { will review the medication prescribed to the patient to identify both potential } \\
\text { underprescribing using the START criteria }{ }^{24} \text { and overprescribing using the STOPP } \\
\text { criteria, }{ }^{1} 13161725 \text { and the Beers criteria. }{ }^{26} \text { An automatic prompt system incorporating the } \\
\text { START \& STOPP criteria and Beers criteria will be implemented }\end{array}$ \\
\hline \multirow[t]{2}{*}{ \#3 } & $10 \mathrm{~min}$ & ECP & Multidisciplinary Meeting \\
\hline & & $\mathrm{P}$ & $\begin{array}{l}\text { In a meeting, the ECP and the P will review all information gathered in step \#1 and \#2. } \\
\text { Subsequently, the information will be reviewed to optimise prescribing (underprescribing/ } \\
\text { overprescribing and misprescribing). If the ECP and P cannot determine the right indication for a } \\
\text { medication (the correct diagnosis), the appropriate medical specialist(s), for example, a } \\
\text { cardiologist, a neurologist and a geriatric psychiatrist will be consulted }\end{array}$ \\
\hline \multirow[t]{2}{*}{ \#4 } & $5 \mathrm{~min}$ & ECP & Pharmacotherapeutical Action Plan \\
\hline & & $\mathrm{P}$ & $\begin{array}{l}\text { The ECP and } \mathrm{P} \text { will register the findings of the review in step \#3 in a pharmaceutical action plan }{ }^{27} \\
\text { and, accordingly, the actions that need to be taken, that is, discontinuation, initiation, dose } \\
\text { adjustment, postponing a decision. This plan will also include tapering-off arrangements to prevent } \\
\text { occurrence of withdrawal symptoms if appropriate }{ }^{28} \text { and appointments about the monitoring of } \\
\text { relapse symptoms and withdrawal effects }\end{array}$ \\
\hline \multirow[t]{2}{*}{ \#5 } & $10 \mathrm{~min}$ & ECP & Implementation \& Interim Evaluation Action Plan \\
\hline & & NS & $\begin{array}{l}\text { The ECP and the NS will further implement the pharmaceutical action plan according to the } \\
\text { agreed schedule. To that end, the nursing staff will be clearly informed. The NS will monitor } \\
\text { adverse withdrawal events when discontinuing medication. The ECP will monitor relapse } \\
\text { symptoms. Furthermore, the patient or his or her representative will be informed about the } \\
\text { medication changes to verify possible objections and to clarify that the aim of the intervention is to } \\
\text { improve the care to nursing home residents. In this phase, the elderly care physician will also } \\
\text { document whether a medication had to be reinstated along with the reasons using so-called } \\
\text { trigger lists }\end{array}$ \\
\hline
\end{tabular}

the patient and their representative in the decision process. $^{27}$ It is very important that a plan is agreed on how to monitor the effects of starting, stopping and changing doses (ie, agree on a scheme of monitoringspecific parameters and responsibilities for the monitoring). This will be documented in the action plan. Again on clinical indication (where necessary and appropriate) specialists will be consulted.

\section{Study parameters}

Main study parameter

The key aim of the 3MR medication reviews is to prevent overprescribing. Hence, the primary endpoint is the difference in proportion of residents who successfully discontinued inappropriate medication between the intervention and control group. Successful discontinuation is defined as discontinuation without relapse of symptoms and without occurrence of withdrawal effects that make it necessary to restart medication. We operationalise successful discontinuation of inappropriate medication as follows: after discontinuation, no new medication within the same Anatomical Therapeutic Chemical (ATC) class or for the same indication will be started at 4-month follow-up (as this would be an indication of relapse of symptoms or moderate to severe withdrawal effects).

\section{Secondary study parameters with regard to optimising} prescribing

- Starting $\geq 1$ medications that should be started on the basis of START criteria. ${ }^{1617}$

- Lowering or increasing of dose. ${ }^{29}$

- The percentage of residents for whom a medication was replaced by a safer alternative. ${ }^{16} 22$

- Exposure to anticholinergic and sedative medication as measured with the DBI. ${ }^{18}$ A higher DBI has been associated with falls in nursing home residents. We will calculate the change in DBI scores from baseline to follow-up using a list of anticholinergic and sedative medication. 
Secondary study parameters with regard to patient outcomes

- Quality of life: as measured with the Dementia Quality of Life Instrument (DQI) ${ }^{31}$ for patients with dementia and the European Quality of Life Questionnaire (EQ-5D-3L), a generic instrument for patients with no dementia. ${ }^{32}$ For the DQI and the EQ-5D-3L, we will calculate the change in scores at follow-up compared with baseline. The DQI and the EQ-5D-3L take approximately 5 min to administer.

- Harm: fall incidents, ${ }^{14}$ 17-19 bone fractures caused by falling, gastrointestinal bleedings, number of visits to outpatient clinics and emergency rooms, hospital admissions, number of visits by medical consultants, that is, physicians who visit the patients in nursing homes, total duration of hospital admissions and mortality risk.

- Cognitive function: as measured with the change in scores on the Severe Impairment Battery (SIB) and the Mini-Mental State Examination (MMSE) at follow-up compared with baseline. The SIB is a standardised objective neuropsychological test of cognitive function, which is specifically designed for patients with advanced dementia. We will use the short form that was developed for nursing home residents ${ }^{33}$ and validated for the Netherlands. ${ }^{34}$ The MMSE is the most widely used brief instrument to assess cognitive function of geriatric patients. ${ }^{35}$ The SIB and the MMSE take approximately $10 \mathrm{~min}$ to administer.

- Neuropsychiatric symptoms: as measured with the change in Neuropsychiatric Inventory (NPI) scores at follow-up compared with baseline. The NPI is a standardised rating instrument of neuropsychiatric symptoms. ${ }^{36}$ We will use the NPI-NH, which was developed and validated for nursing home residents. ${ }^{37}$ The NPI-NH takes on average 10 min to administer.

- Laboratory results: All biochemical, haematology and microbiology laboratory examinations that are indicative of organ malfunction and/or recurrence of conditions for which medicines were initially prescribed.

\section{Costs}

To enable a cost-effectiveness analysis, all relevant costs of conducting the medication reviews and healthcare utilisation will be estimated following the costeffectiveness study conducted by Patterson et al. ${ }^{15}$ Costs will be based on legal national healthcare and regional laboratory tariffs and include:

Medication review expenditures

- Training of elderly care physician, nursing staff and pharmacist.

- Salary costs of elderly care physician, nursing staff and pharmacist involved with carrying out the medication reviews including implementation by elderly care physician.

Healthcare utilisation expenditures

- Salary costs: medical consulting time estimated from the patient's medical status including consultations by elderly care physicians, all other physicians including medical consultants and other paramedical care professionals (physiotherapist, speech and language therapist, acoustician, optometrist, dentist, dietitian, specialised nurse, pharmacist, podiatrist and nursing home psychologist).

- Medication costs: of all medication prescribed.

- Laboratory examinations: of all biochemical, haematology and microbiology laboratory examinations.

- Additional costs: of total duration and number of visits to emergency room, outpatient clinics and hospital admissions.

\section{Covariates}

Covariates at the nursing home level as well as on the nursing home resident's level will be measured and include:

Nursing home characteristics

Prescribing information, type of ward (for nursing home residents with dementia vs those with physically disabling conditions) and number of residents per ward. Elderly care physicians

- Number of years of working experience as physician in the fields of elderly care, geriatrics and geriatric psychiatry.

- Amount of training with regard to geriatric pharmacotherapy, clinical pharmacology, polypharmacy, inappropriate prescribing and medication review methodology.

Nursing home residents' characteristics

- Demographic characteristics: sex, age, educational level, marital status.

- Clinical characteristics: length of stay in nursing home, number of prescribed medicines, medical conditions and a revised Charlson's comorbidity index. ${ }^{38}$

\section{Randomisation, blinding and intervention allocation}

Randomisation will occur at the level of the elderly care physician and his or her ward such that half of the elderly care physicians will be randomly assigned to perform the 3MR (the intervention condition) and half will perform usual care (the control condition). This will be done to avoid cross-contamination. Randomisation will be performed by one of the researchers who is not involved in collecting the data from participants. Because of the relatively small number of elderly care physicians $(\mathrm{N}=30)$, they will be matched first on relevant characteristics including supplying pharmacy, healthcare organisation, type of ward(s) (for residents with dementia vs those with physically disabling conditions or both), and number of residents of their ward(s). Based on this matching procedure, pairs of elderly care physicians who are most alike will be made. Subsequently, within each pair, one physician will be randomly assigned to the intervention condition and one physician will be randomly assigned to the control condition. ${ }^{39}$ Random assignment will be established using the random variable function of SPSS. As clinicians will perform the $3 \mathrm{MR}$ medication review, 
they cannot be kept blind to the patient's allocation to the experimental condition (receiving the medication review) versus the control condition (usual care). Nursing home residents, their legal representatives and assessors of the study parameters will, however, be kept blind to intervention allocation as far as possible. The study is therefore a single blind study.

\section{Quality of data collection}

Data will be collected in a standardised manner using data collection forms. A trained neuropsychologist will assess residents' cognitive function using objective and validated neuropsychological tests (see above 'Study parameters' section). Neuropsychiatric symptoms and quality of life will be assessed by a member of the staff using validated questionnaires (see above 'Study parameters' section). To counteract occurrence of missing data, completeness of data for each individual resident will be monitored using standardised progress forms. Follow-up data will be collected 4 months later. Although this is relatively soon, this will be done because of the high mortality and morbidity risk of nursing home residents. Data will be entered twice by two research assistants in two independent data files. Discrepancies between the files will be checked against hardcopy and source data and errors will be corrected accordingly.

\section{Statistical analysis}

Descriptive results will be presented at the patient and the ward level. Potential bias will be examined in the following respects. First, a non-response analysis will be performed to examine whether male or female and younger or older residents were more likely to participate. Second, extent of differential attrition will be assessed by examining whether residents who were lost to follow-up significantly differed on baseline characteristics from residents who were retained at follow-up. Successful discontinuation of inappropriate medication and secondary study parameters will be treated as dependent variables in generalised linear mixed models. This type of analysis will be employed to account for dependence of data (nursing home residents within wards). To that end, a random intercept and a random slope at the level of wards will be entered into the linear mixed model. If randomisation is successful, no adjustment for covariates will be necessary. In that case, only the effect of condition (intervention versus control condition) will be examined. Consistency of findings will be assessed with sensitivity analyses. First, we will adopt an intention-to-treat approach in which nursing home residents who underwent a medication review but who were lost to follow-up will be included in the analysis and counted as if medicines could not be successfully stopped ('intention to treat'). Second, in a per-protocol analysis, we will include only those nursing home residents who actually received the intervention and who have complete data. All statistical tests will be one sided. $\mathrm{p}$ Values $\leq 5 \%$ will be considered significant.

\section{Cost-effectiveness analysis}

The cost-effectiveness of the 3MR will be examined by calculating the Incremental Cost Effectiveness Ratio (ICER). Two different ICERs will be calculated. The first ICER will reflect the difference in the mean costs (in US\$) between the intervention and control group divided by the difference in the percentage of residents who successfully discontinue medication in the intervention and control group. The second ICER will reflect the difference in the mean costs (in US\$) between the intervention and control group divided by the difference in estimated quality-adjusted life-years (QALYs) in the intervention and control group. QALYs will be estimated using the utilities of health states on the DQI on baseline and follow-up. Uncertainty in the point estimate of the ICER will be investigated using 95\% CIs obtained with non-parametric bootstrapping.

\section{Study procedures}

The flow chart of figure 1 provides a schematic overview of the study phases along with the participant flow, for example, how many participants decline participation or are lost to follow-up. Different wards will participate in a sequential manner to the study.

\section{ETHICS AND DISSEMINATION}

The study will be conducted in accordance with the Declaration of Helsinki regarding the Ethical Principles for Medical Research Involving Human Subjects (amended by the 64th World Medical Association's General Assembly, Fortaleza, Brazil, October 2013). All nursing home residents will be asked to provide written informed consent (IC). In case a resident is incapacitated, her or his legal representative will be asked to consent instead. Regardless of IC, signs expressed by nursing home residents, which might be indicative of objection, will be carefully monitored and will be deemed an instant reason for discontinuation of testing.

Furthermore, after careful weighting of the benefits and harms, we are confident that systematic medication reviews have much potential for nursing home residents (as outlined above). At the same time, the risk of occurrence of (serious) adverse events related to study participation will be deemed highly unlikely. Systematic medication reviews will be carried out by the treating elderly care physician and the pharmacist and the final responsibility to make definite treatment decisions will rest with the treating elderly care physician.

Data will be confidentially handled and stored. To assure confidentiality, research data and residents' personal data will be stored in two different files. Data records from both files will be linked with an identification number that cannot be traced to the individual residents and their personal characteristics, for example, year of birth. The file with residents' personal data will be password protected and will be safeguarded by the 


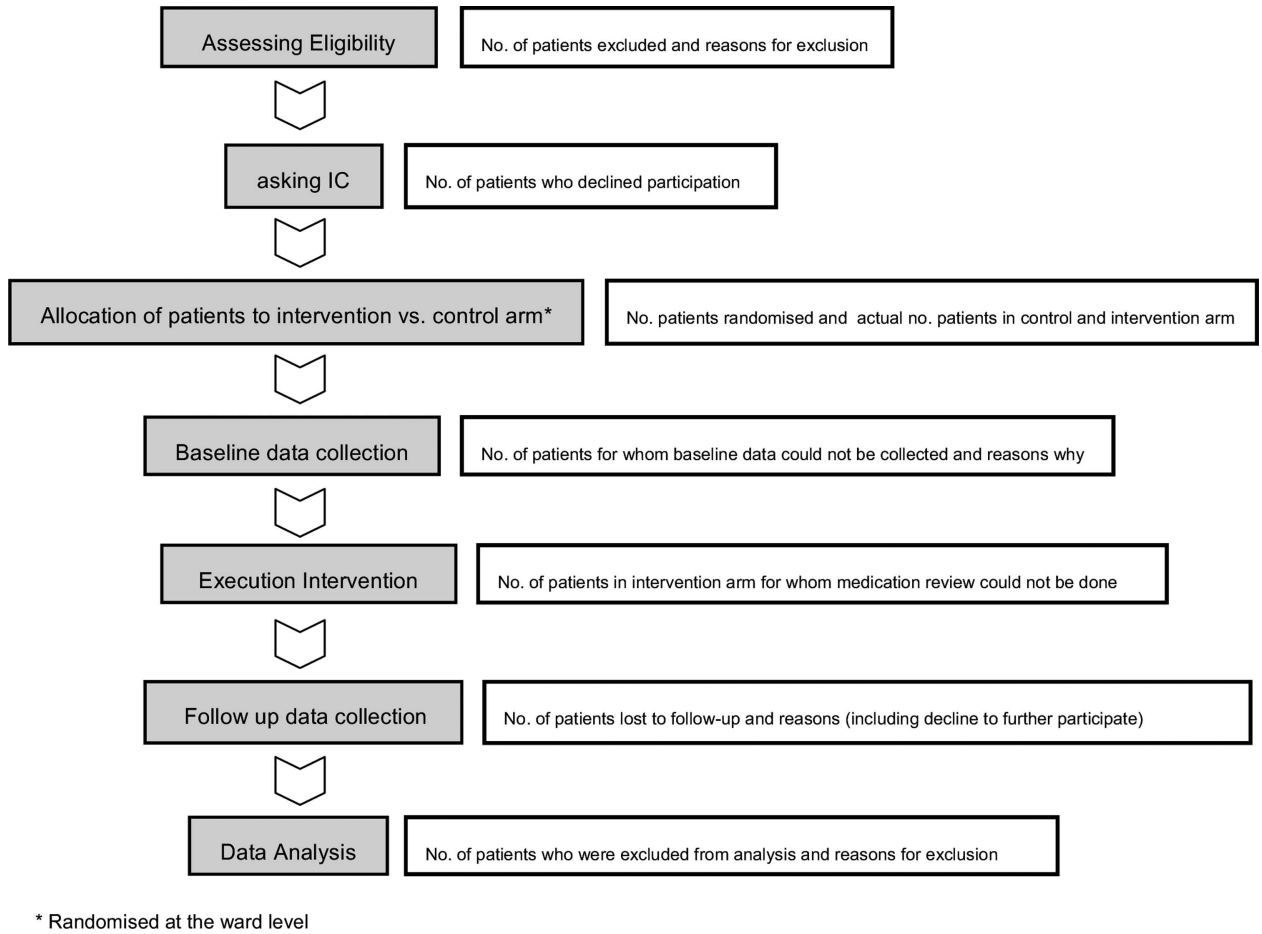

Figure 1 Flow chart of study phases (IC, informed consent).

investigators. To avoid scientific fraud or misconduct, all investigators will have full access to the data.

Finally, study results will be published in peer-reviewed journals and in relevant news channels for clinicians. In addition, this study has been registered at http:// www.ClinicalTrials.gov (trial registration number: NCT01876095). Duplicate publication will be avoided.

In addition to publication of study results, a practical toolkit will be constructed to carry out the $3 \mathrm{MR}$ medication reviews.

\section{Author affiliations}

${ }^{1}$ Department of Pharmacy, Unit of Pharmacotherapy and Pharmaceutical Care, Groningen Research Institute of Pharmacy, Faculty of Mathematics and Natural Sciences, University of Groningen, Groningen, The Netherlands ${ }^{2}$ Department of General Practice, University of Groningen, University Medical Centre Groningen, Groningen, The Netherlands

${ }^{3}$ Hospital Pharmacy, Diaconessenhuis, Meppel, The Netherlands ${ }^{4}$ Hospital Pharmacy, Wilhelmina Ziekenhuis Assen, Assen, The Netherlands ${ }^{5}$ Department of Clinical Pharmacy and Toxicology, Martini Ziekenhuis Groningen, Groningen, The Netherlands

${ }^{6}$ Department of Pharmacy, Unit of Pharmaco-epidemiology and Pharmacoeconomics, Groningen Research Institute of Pharmacy, Faculty of Mathematics and Natural Sciences, University of Groningen, Groningen, The Netherlands

Contributors HW, FB, PN, JB, WMB, HM, J-GM, MJP, SUZ and KT contributed to the study conception and design. HW, FB, PN, JB, WMB, HM, J-GM, LW-V, TB, JVD, SUZ and KT contributed to the intervention development. HW, EHQ, FB, MJP, SUZ, and KT reviewed the study parameters and contributed to the analysis plan. HW, FB, PN, SUZ and KT obtained ethical approval. HW, EHQ, FB, J-GM, MJP, SUZ and KT drafted the manuscript. HW, EHQ, FB, PN, JB, WMB, HM, J-GM, LW-V, TB, JvD, MJP, SUZ and KT revised and approved the final manuscript.

Funding This work was supported by the Netherlands Organisation for Health Research and Development (ZonMw; grant number 80-83600-98-10176).
Competing interests None.

Ethics approval Medical Ethical Committee of the University Medical Centre Groningen (protocol number NL48091.042.14; in Dutch: Medisch Ethische Toetsingscommissie van het Universitair Medisch Centrum Groningen [METc UMCG]).

Provenance and peer review Not commissioned; peer reviewed for ethical and funding approval prior to submission.

Open Access This is an Open Access article distributed in accordance with the Creative Commons Attribution Non Commercial (CC BY-NC 4.0) license, which permits others to distribute, remix, adapt, build upon this work noncommercially, and license their derivative works on different terms, provided the original work is properly cited and the use is non-commercial. See: http:// creativecommons.org/licenses/by-nc/4.0/

\section{REFERENCES}

1. Gallagher $P$, Barry $P, O$ 'Mahony $D$. Inappropriate prescribing in the elderly. J Clin Pharm Ther 2007;32:113-21.

2. Ruggiero C, Dell'Aquila G, Gasperini B, et al. Potentially inappropriate drug prescriptions and risk of hospitalisation among older, Italian, nursing home residents: the ULISSE project. Drugs Aging 2010;27:747-58.

3. Perri M, Menon AM, Deshpande AD, et al. Adverse outcomes associated with inappropriate drug use in nursing homes. Ann Pharmacother 2005;39:405-11.

4. Jano E, Aparasu RR. Healthcare outcomes associated with Beers' criteria: a systematic review. Ann Pharmacother 2007;41:438-47.

5. Bergh S, Selbaek G, Engedal K. Discontinuation of antidepressants in people with dementia and neuropsychiatric symptoms (DESEP study): double blind, randomised, parallel group, placebo controlled trial. BMJ 2012;344:e1566.

6. Iyer S, Naganathan V, McLachlan AJ, et al. Medication withdrawal trials in people aged 65 years and older: a systematic review. Drugs Aging 2008;25:1021-31.

7. Molander L, Lovheim H, Norman $\mathrm{T}$, et al. Lower systolic blood pressure is associated with greater mortality in people aged 85 and older. J Am Geriatr Soc 2008;56:1853-9.

8. Ballard C, Margallo-Lana M, Juszczak E, et al. Quetiapine and rivastigmine and cognitive decline in Alzheimer's disease: randomised double blind placebo controlled trial. BMJ 2005;330:874 
9. Seitz DP, Gill SS, Herrmann N, et al. Pharmacological treatments for neuropsychiatric symptoms of dementia in long-term care: a systematic review. Int Psychogeriatr 2013;25:185-203.

10. Ballard C, Lana MM, Theodoulou M, et al. A randomised, blinded, placebo-controlled trial in dementia patients continuing or stopping neuroleptics (the DART-AD trial). PLoS Med 2008:5:e76.

11. Devanand DP, Mintzer J, Schultz SK, et al. Relapse risk after discontinuation of risperidone in Alzheimer's disease. N Engl J Med 2012;367:1497-507.

12. Garfinkel D, Zur-Gil S, Ben-Israel J. The war against polypharmacy: a new cost-effective geriatric-palliative approach for improving drug therapy in disabled elderly people. Isr Med Assoc J 2007;9:430-4.

13. Christensen D, Trygstad T, Sullivan R, et al. A pharmacy management intervention for optimizing drug therapy for nursing home patients. Am J Geriatr Pharmacother 2004;2:248-56.

14. Patterson SM, Hughes CM, Crealey G, et al. An evaluation of an adapted U.S. model of pharmaceutical care to improve psychoactive prescribing for nursing home residents in Northern Ireland (Fleetwood Northern Ireland study). J Am Geriatr Soc 2010;58:44-53.

15. Patterson SM, Hughes CM, Cardwell C, et al. A cluster randomised controlled trial of an adapted U.S. model of pharmaceutical care for nursing home residents in Northern Ireland (Fleetwood Northern Ireland study): a cost- effectiveness analysis. J Am Geriatr Soc 2011:59:586-93.

16. Loganathan M, Singh S, Franklin BD, et al. Interventions to optimise prescribing in care homes: systematic review. Age Ageing 2011:40:150-62.

17. Forsetlund L, Eike MC, Gjerberg E, et al. Effect of interventions to reduce potentially inappropriate use of drugs in nursing homes: a systematic review of randomised controlled trials. BMC Geriatr 2011;11:1-18.

18. Wilson NM, Hilmer SN, March LM, et al. Associations between drug burden index and falls in older people in residential aged care. J Am Geriatr Soc 2011;59:875-80.

19. Cornege-Blokland E, Kleijer BC, Hertogh CM, et al. Reasons to prescribe antipsychotics for the behavioural symptoms of dementia: a survey in Dutch nursing homes among physicians, nurses, and family caregivers. J Am Med Dir Assoc 2012;13:80.e1,80.e6.

20. Wetzels RB, Zuidema SU, de Jonghe JF, et al. Prescribing pattern of psychotropic drugs in nursing home residents with dementia. Int Psychogeriatr 2011;23:1249-59.

21. Hughes CM, Goldie R. "I just take what I am given": adherence and resident involvement in decision making on medicines in nursing homes for older people: a qualitative survey. Drugs Aging 2009;26:505-17.

22. Garfinkel D, Mangin D. Feasibility study of a systematic approach for discontinuation of multiple medications in older adults: addressing polypharmacy. Arch Intern Med 2010;170:1648-54.

23. Zuidema SU, de Jonghe JF, Verhey FR, et al. Psychotropic drug prescription in nursing home patients with dementia: influence of environmental correlates and staff distress on physicians' prescription behaviour. Int Psychogeriatr 2011;23:1632-9.
24. Gallagher P, Baeyens JP, Topinkova E, et al. Inter-rater reliability of STOPP (screening tool of older persons' prescriptions) and START (screening tool to alert doctors to right treatment) criteria amongst physicians in six European countries. Age Ageing 2009; 38:603-6.

25. Gallagher P, Ryan C, Byrne S, et al. STOPP (Screening Tool of Older Person's Prescriptions) and START (Screening Tool to Alert doctors to Right Treatment). Consensus validation. Int J Clin Pharmacol Ther 2008;46:72-83.

26. American Geriatrics Society 2012 Beers Criteria Update Expert Panel. American geriatrics society updated beers criteria for potentially inappropriate medication use in older adults. J Am Geriatr Soc 2012;60:616-31.

27. Finkers F, Maring JG, Boersma F, et al. A study of medication reviews to identify drug-related problems of polypharmacy patients in the Dutch nursing home setting. J Clin Pharm Ther 2007;32:469-76.

28. Bain KT, Holmes HM, Beers MH, et al. Discontinuing medications: a novel approach for revising the prescribing stage of the medication-use process. J Am Geriatr Soc 2008;56:1946-52.

29. Spinewine A, Schmader KE, Barber N, et al. Appropriate prescribing in elderly people: how well can it be measured and optimised? Lancet 2007;370:173-84.

30. Zermansky AG, Alldred DP, Petty DR, et al. Clinical medication review by a pharmacist of elderly people living in care homesrandomised controlled trial. Age Ageing 2006;35:586-91.

31. Schölzel-Dorenbos CJ, Arons AM, Wammes JJ, et al. Validation study of the prototype of a disease-specific index measure for health-related quality of life in dementia. Health Qual Life Outcomes 2012;10:1-11

32. Herdman M, Gudex C, Lloyd A, et al. Development and preliminary testing of the new five-level version of EQ-5D (EQ-5D-5 L). Qual Life Res 2011;20:1727-36.

33. Saxton J, Kastango KB, Hugonot-Diener L, et al. Development of a short form of the severe impairment battery. Am J Geriatr Psychiatry 2005;13:999-1005

34. De Jonghe JF, Wetzels RB, Mulders A, et al. Validity of the severe impairment battery short version. $J$ Neurol Neurosurg Psychiatry 2009;80:954-9.

35. Folstein MF, Folstein SE, McHugh PR. Mini-mental state: a practical method for grading the cognitive state of patients for the clinician J Psychiatr Res 1975;12:189-98.

36. Cummings JL, Mega M, Gray K, et al. The neuropsychiatric inventory: comprehensive assessment of psychopathology in dementia. Neurology 1994:44:2308-14.

37. Kat MG, de Jonghe JF, Aalten P, et al. Neuropsychiatric symptoms of dementia: psychometric aspects of the Dutch neuropsychiatric inventory (NPI). Tijdschr Gerontol Geriatr 2002;33:150-5.

38. Quan H, Li B, Couris CM, et al. Updating and validating the Charlson comorbidity index and score for risk adjustment in hospital discharge abstracts using data from 6 countries. Am J Epidemiol 2011;173:676-82.

39. Schulz KF, Grimes DA. Generation of allocation sequences in randomised trials: chance, not choice. Lancet 2002;359:515-19. 